\title{
THE MANEUVER PLANNING PROCESS FOR THE MICROWAVE ANISOTROPY PROBE (MAP) MISSION
}

\author{
Michael A. Mesarch* \& Stephen F. Andrews ${ }^{\dagger}$ \\ National Aeronautics and Space Administration \\ Goddard Space Flight Center, Code 572 \\ Greenbelt, MD 20771
}

\begin{abstract}
The Microwave Anisotropy Probe (MAP) mission utilized a strategy combining highly eccentric phasing loops with a lunar gravity assist to provide a zero-cost insertion into a Lissajous orbit about the Sun-Earth/Moon L2 point. Maneuvers were executed at the phasing loop perigees to correct for launch vehicle errors and to target the lunar gravity assist so that a suitable orbit at L2 was achieved. This paper will discuss the maneuver planning process for designing, verifying, and executing MAP's maneuvers. This paper will also describe how commercial off-the-shelf (COTS) tools were used to execute these tasks and produce a command sequence ready for upload to the spacecraft. These COTS tools included Satellite Tool Kit, MATLAB, and Matrix-X.
\end{abstract}

\section{Introduction}

The MAP mission was launched on June 30, 2001 from Cape Canaveral on a Delta-II 7425-10 expendable launch vehicle. The spacecraft was separated into a highly elliptical orbit with an inclination of $28.7^{\circ}$ and a C3 energy of $-2.6 \mathrm{~km}^{2} / \mathrm{s}^{2}$. MAP remained in this "phasing" orbit for three full loops before encountering the Moon and receiving a gravity assist to the Sun-Earth/Moon L2 Lagrange point (1.5 million kilometers from the Earth, in the direction opposite the Sun). From its Lissajous orbit about L2, MAP will measure the temperature fluctuations of the cosmic microwave background, the radiant heat left over from the Big Bang. MAP is a follow-on mission to NASA's Cosmic Background Explorer (launched in 1989).

After separation from the launch vehicle, MAP was required to perform several perigee maneuvers during the phasing loop portion of the mission in order to accurately target the lunar encounter. Overall, seven maneuvers were planned to occur before the lunar encounter - three perigee maneuvers, three engineering burns (which occurred at the three phasing loop apogees), and a final perigee correction maneuver (PfCM) 18 hours after the final perigee. After the lunar encounter, two mid-course correction maneuvers (MCCM) were required to fine-tune the trajectory. The end result of these maneuvers was to achieve a Lissajous orbit that met all MAP's mission requirements'. Since the zero-cost insertion into the Lissajous orbit, two stationkeeping maneuvers (SK1 \& SK2) have been performed to keep MAP orbiting around L2. Future stationkeeping maneuvers will be required every three months for the life of the mission (two years, with a goal of four years). A picture of MAP's trajectory can be seen in Figure 1. Complex planning and coordination was needed to ensure the success of these maneuvers as the maneuver products crossed many disciplines (Table 1). While the process described herein applies to all maneuvers, the following discussion will be concerned with the time-critical perigee maneuvers and the PfCM.

Table 1: MAP Maneuver Team \& Responsibilities

\begin{tabular}{|l|l|}
\hline \multicolumn{1}{|c|}{ MAP Subsystem } & Maneuver Planning Activities \\
\hline Trajectory Design & $\checkmark$ Defined time, magnitude, and attitude tor all maneuvers \\
& $\checkmark$ Verified maneuver against mission constraints \\
\hline Attitude Control System & $\checkmark$ Executed maneuvers in various simulators prior to upload to MAP \\
(ACS) & $\checkmark$ Computed attitude control dutycycles for thrusters \\
& $\checkmark$ Verified maneuver against attitude constraints \\
\hline Propulsion & $\checkmark$ Provided state of propulsions system (tank pressure, tank temperature, fuel remaining) \\
& $\checkmark$ Monitored performance of propulsion system \\
\hline Navigation & $\checkmark$ Calculated orbit determination solution \\
& $\checkmark$ Delivered acquisition data to tracking assets \\
\hline Thermal & $\checkmark$ Evaluated attitude profile for solar intrusion onto MAP's instrument \\
\hline Power & $\checkmark$ Evaluated attitude profile to ensure sufficient power for maneuver \\
\hline Ground Systems & $\checkmark$ Scheduled tracking data periods with DSN and TDRS \\
& $\checkmark$ Generated attitude profile and command sequence for upload to MAP \\
\hline MAP Systems Engincer & $\checkmark$ Provided oversight of entire process \\
& $\checkmark$ Correlated solar activity levels with threat potential to MAP during maneuvers \\
\hline
\end{tabular}

* Flight Dynamics Engineer, Guidance, Navigation, and Control Center, Senior Member AIAA

Attitude Control System Engineer, Guidance, Navigation, and Control Center 


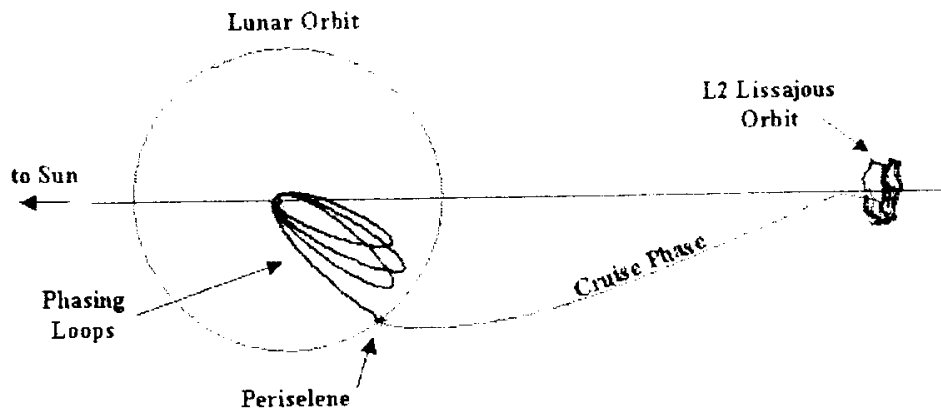

Figure 1: MAP Trajectory to L2 (Solar Rotating Coordinates)

\section{MAP Propulsion Svstem}

The MAP spacecraft carries a mono-propellant hydrazine system with a beginning-of-life, full tank load of $72.57 \mathrm{~kg}$ of fuel. Connected to the tank are eight, one-pound (4.45 Newton) thrusters which are grouped into three primary firing modes: $+X,+Z$, and $Z$ (referenced to the body axes). The $+X$ thrusters consist of thrusters $5 \& 6$ (oriented along the $+X$ body axis) and thrusters $7 \& 8$ (oriented $15^{\circ}$ off of the $+X$ axis, towards $+Z$ ). Due to the orientation of thrusters 7 $\& 8$, performing maneuvers utilizing thrusters $5,6,7, \&$ 8 requires pitching the spacecraft $7.5^{\circ}$ (about the $+Y$ axis) to align the resultant thrust vector along the desired thrust direction. The $+X$ thrusters are used for the perigee maneuvers and the PfCM. A $+Z$ maneuver uses thrusters $3 \& 4$ (oriented $30^{\circ}$ off of the $Z$-axis) and a $-Z$ maneuver uses thrusters $1 \& 2$ (orientated $10^{\circ}$ off of the $Z$-axis, towards $-X)$. The $\pm Z$ thrusters are used for the MCCM and SK maneuvers. During any maneuver, all thrusters are included in the control loop to maintain pointing and stability. The layout of MAP's thrusters can be seen in Figure 2. A more detailed discussion of MAP's propulsion system can be found in Reference 2.
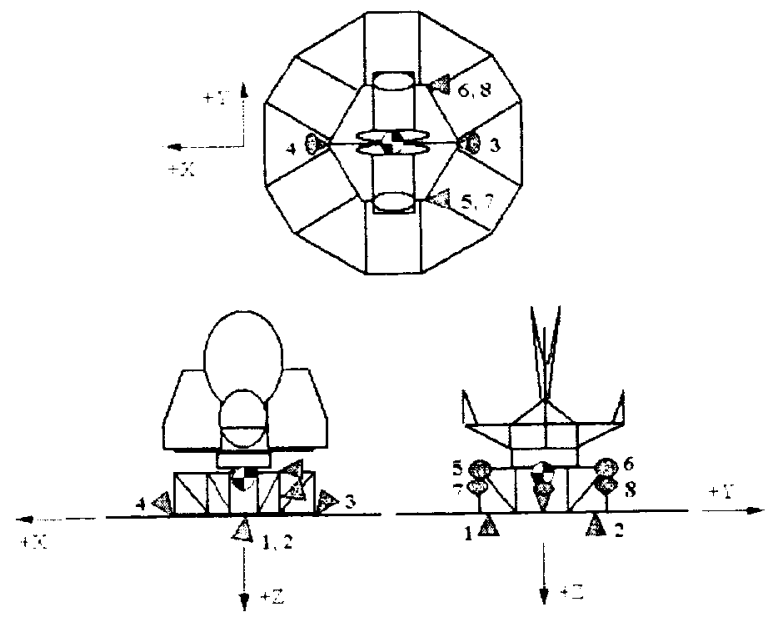

Figure 2: MAP Thruster Layout

\section{Maneuver Planning Process}

As mentioned above, MAP's maneuver planning required input and verification from many different disciplines. At the same time, the process also involved bringing together many different pieces of software, many of which were commercial off-the-shelf (COTS) products. Satellite Took Kit's (STK) Astrogator module, developed by Analytical Graphics, Inc., was used as the major tool for MAP's trajectory design activities. Astrogator was used for the high-fidelity trajectory modeling and maneuver planning. STK's Visualization Option (VO) was used to represent the trajectory in 3-dimensional space while viewing such objects as sensor cones and attitude orientation. MATLAB, developed by The MathWorks Inc., was used to create the Quaternion Generator (QuatGen) tool for processing the maneuver attitude. MATLAB was also used extensively as a data reduction tool by the trajectory design team. Matrix-X, originally developed by Integrated Systems but now distributed by The MathWorks Inc., was used to create $\mathrm{HiFi}$, a high fidelity, software-only simulator of the MAP attitude control system. The final major component was FlatSat, a real-time hardware-in-the-loop simulator, which used engineering test units (ETU's), ground system terminals, and MAP flight software loaded to the ETU's to simulate operations on the spacecraft. A simplified form of the process is shown in Figure 3. The major inputs (e.g. impulsive maneuver plan, orbit determination state, and the propulsion state) were passed into Astrogator for an initial finite maneuver plan. Furthermore, great care was taken to make sure all components of the process used the same database information. This information included thruster unit vectors, propulsion blowdown parameters, etc. Output from this plan was passed through QuatGen to create a command quaternion table (CQT) - a time history of the desired attitude for the maneuver. This data was then passed into both simulators, $\mathrm{HiFi}$ and FlatSat, to simulate the maneuvers. The difference in these simulators is part speed and part fidelity. HiFi performs a fast simulation of the maneuver as it pertains to the 
ACS subsystem. FlatSat, on the other hand, is a realtime simulator that was used to test all of the maneuver commands on the ground before they were uploaded to the spacecraft. After each simulation, Astrogator was used to "close the loop", processing outputs from each simulator to verify that no significant change to the nominal trajectory occurred. The results of these simulations were made available to the remaining subsystems for concurrence. Prior to each maneuver, a command authorization meeting (CAM) was held during which the maneuver plan was examined in detail. After it was determined that all subsystems were in agreement with the plan, each subsystem lead signed-off on the final maneuver plan. The detailed version of this process is shown in Figure 4 . The following sections of the paper will discuss these different tools and how they were used in this process.

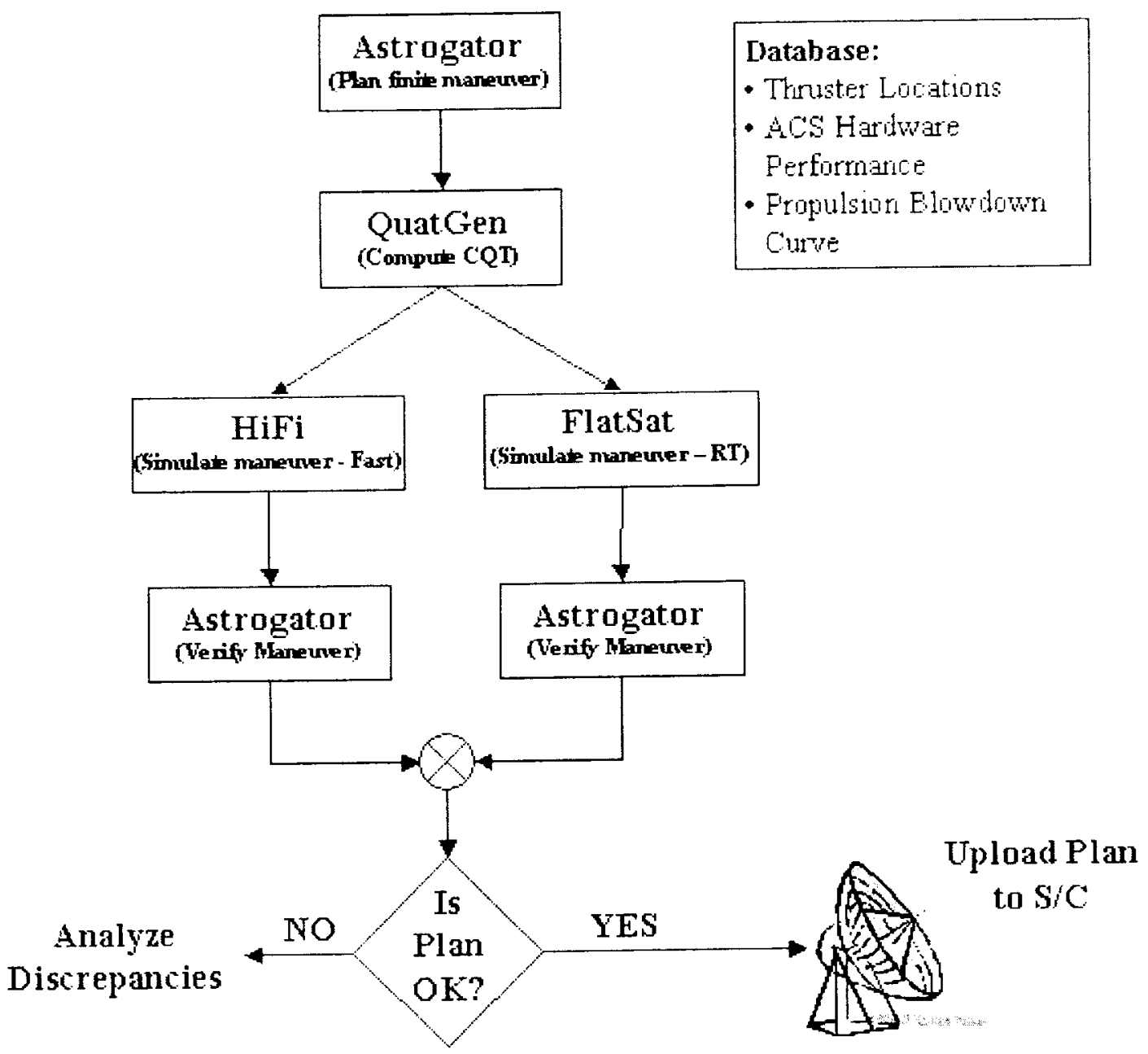

Figure 3: Maneuver Planning Process 


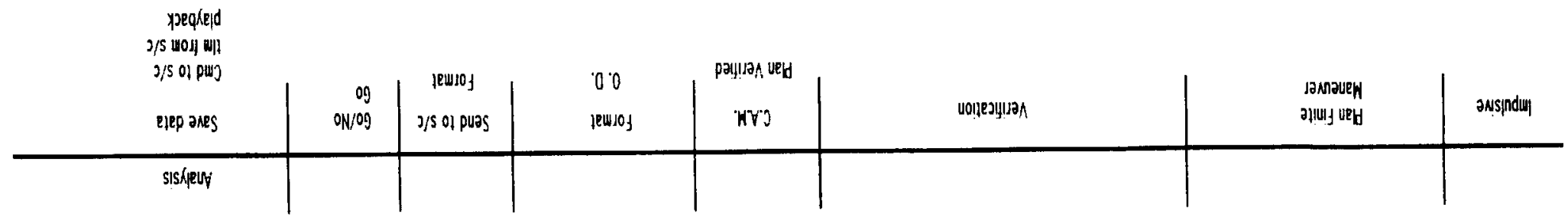

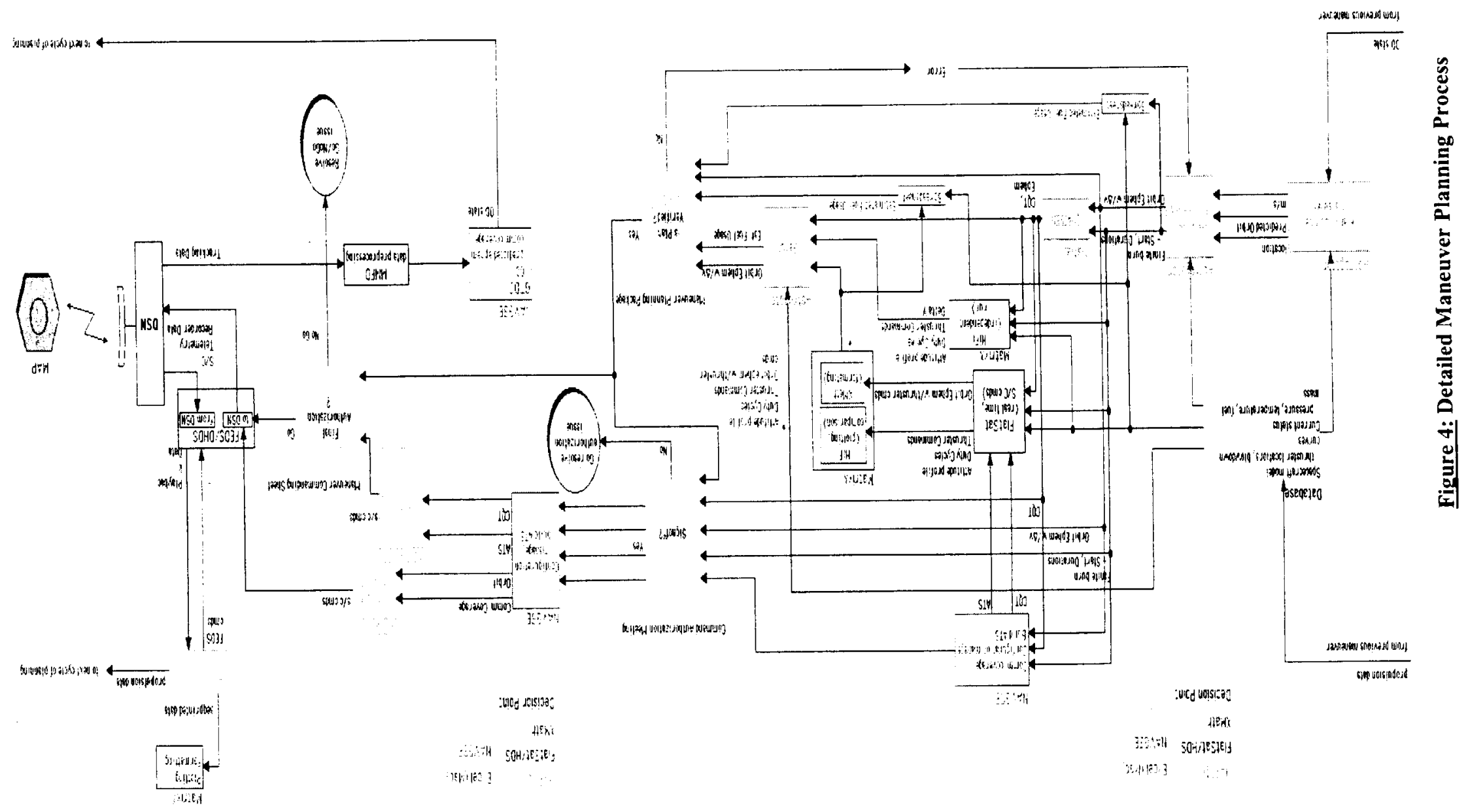


The timing for this process started with the delivery of the orbit determination solution 23 hours prior to maneuver ignition (M-23). The trajectory team used the current orbit and propulsion states to produce a preliminary finite maneuver plan. This plan was used to deliver products at $\mathrm{M}-20$ for the HiFi and FlatSat simulation. The results from the $\mathrm{HiFi}$ and FlatSat simulations were completed at M-19 and M-16 hours, respectively. After verification of the simulations by the trajectory design team, data was sent to the other subsystems for validation at M-13 hours. Typically, the command authorization meeting (CAM) was held at $\mathrm{M}$ 11 hours, at which time the maneuver plan was discussed with all subsystem representatives present. The Automatic Time Sequence (ATS), the list of commands necessary to perform the maneuver (including the CQT), was uploaded to MAP following the successful completion of the CAM. After verification of successful transmission to MAP, the ATS was enabled. The first commands from the ATS were typically executed at M-2 hours.

\section{STK/Astrogator}

STK's Astrogator module was the primary tool used for MAP's trajectory design. The MAP team used Astrogator version 4.1.1 on a Windows NT platform for all of the trajectory design and maneuver planning activities. The maneuver design process began with the receipt of an orbit determination state from the Navigation team. Tracking data from the Deep Space Network's (DSN) ground station was processed using the Goddard Trajectory Determination System (GTDS) to produce an STK-formatted ephemeris file. An orbit state from the ephemeris file was ingested into STK's Astrogator module to use as the initial state. The Propulsion engineer was responsible for supplying the current propulsion state - fuel remaining and tank pressure. The tank temperature was not used as it was determined to be a second order effect. Important database information was also included as inputs. This included thruster unit vectors, measured prior to launch, along with the thruster performance, in terms of force (Newtons) and specific impulse (Isp, in seconds). The thruster performance was delivered in the form of blowdown curves as a function of tank pressure. For MAP, it was decided that each thruster would keep the same performance level and calibration would be performed on the entire system, as opposed to calibrating each thruster. Other database information included the MAP dry mass, tank volume, fuel density, solar radiation pressure area, \& coefficient of reflectivity $\left(C_{R}\right)$. With MAP's mission requirement of perigee heights greater than $500 \mathrm{~km}$, atmospheric drag during the phasing loop perigees was not considered. In fact, MAP's June $30^{\text {th }}$ launch date and a nominal launch vehicle insertion ensured that no perigee height was less than $1000 \mathrm{~km}$.

After inclusion of the orbit determination and propulsion states, the impulsive maneuver plan in Astrogator was converted to a finite maneuver plan. All MAP trajectory planning was performed using impulsive maneuvers. The impulsive to finite transformation only occurred in preparation for executing a maneuver. For any finite maneuver, a "thruster set" ( $+\mathrm{X},+\mathrm{Z}$, or $-\mathrm{Z}$ as defined above), coordinate frame, Euler angle rotation, start time, and duration were selected for the particular maneuver. Regardless of the maneuver type, all maneuvers were planned such that the Sun remained in MAP's digital sun sensor (DSS) field of view. The DSS was used as a backup rate source in the event of an Inertial Reference Unit (IRU, or gyro) failure. The DSS field of view is formed from two sensors mounted on the $+Z$ side of the spacecraft. Each sensor has a $64^{\circ} \times 64^{\circ}$ rectangular field of view. The sensors were mounted $\pm 29.5^{\circ}$ off of the $+Z$ axis to create a usable field of view of $50^{\circ}$ by $110^{\circ}$. The $\pm Z$ maneuvers keep the Sun in the field of view by default, since these maneuvers are performed at an attitude where the $+Z$-axis is $19^{\circ}$ off of the MAPSun line - the nominal attitude for stationkeeping maneuvers. The $+\mathrm{X}$ maneuvers were slightly different. These maneuvers were planned in a "Velocity-Sun" frame. First, the MAP body $X$-axis is aligned with the velocity vector. Then, the $Z$-axis is orientated towards the Sun while staying in the plane made by the velocity vector and the MAP-Sun vector. Then, MAP was pitched $7.5^{\circ}$ about the $Y$-axis to align the resultant $+X$ thrust vector with the velocity vector (Figure 5), as displayed using STK VO. In this alignment, the Sun remains in the center of the short axis of the DSS field of view while it travels along the long axis of the field of view. During the first perigee (P1) maneuver, the Sun traveled through more than half of the field of view. Shortly after launch, MAP instrument personnel changed the maneuver requirement limiting the DSS FOV to $50^{\circ}$ by $90^{\circ}$ due to the potential for Sun impingement on the instrument's main reflector at the extremes of the field of view. The QuatGen program was modified to handle this late change. Regardless, the trajectory team analysts were able to model this field of view by creating a sensor object in STK and attaching it to the vehicle. Viewing the sensor dynamics during maneuvers in STK's VO module was indispensable in regards to analyzing the maneuver. 


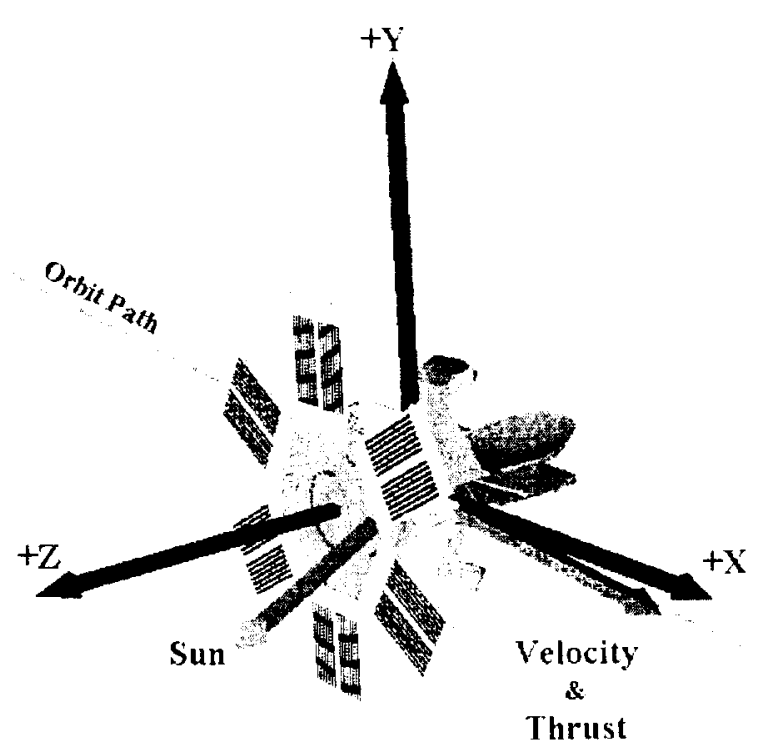

Figure 5: MAP Attitude for $+X$ Maneuvers

Once the attitude was properly set, a differentialcorrection targeting scheme was executed to determine the optimal start time and duration for the burn. The targeting was performed using two control variables (start time and duration) and two goals (lunar encounter parameters). After the scheme converged, product generation for the attitude simulations could begin. One output of this process was a one-line file containing data for the HiFi and FlatSat simulations. This file contained the maneuver start time, the maneuver duration, the maneuver average thrust ( $\mathrm{HiFi}$ did not have a blowdown model for thrust), the spacecraft mass prior to ignition, and the tank pressure prior to ignition. All unit issues were resolved beforehand and were documented in an interface control document (ICD) ${ }^{3}$. The remaining products were created using QuatGen a MATLAB utility used to output the attitude quaternions to control to during the maneuver. All $+\mathrm{X}$ maneuvers were initially planned using only thrusters 5 , $6,7, \& 8$ with a dutycycle of $100 \%$. The $\mathrm{HiFi}$ and FlatSat simulations modeled the attitude dynamics and would provide the appropriate thruster dutycycles for all eight thrusters. This included off-pulsing by the $+X$ thrusters and on-pulsing by thrusters one through four. Similarly, the $\pm Z$ maneuvers were initially planned using only thrusters $3 \& 4$ or $1 \& 2$, respectively. The attitude simulations provided the appropriate dutycycles for those maneuvers as well.

After the HiFi and FlatSat simulations were performed, Astrogator was used to close the loop by feeding results from those simulations back into trajectory plan and examining the results. The simulation results included the dutycycle thrusting of all eight thrusters, an attitude history file, and an adjusted burn duration. The attitude history contained the estimated quaternions that the ACS controlled to during the simulation. As MAP needs to follow the velocity vector during the perigee burn, the ACS must continually re-point the spacecraft. This attitude history file simulates MAP's "crab-walk" through the perigee maneuver. The adjusted burn duration results from the programming of the ACS. For example, during a $+X$ maneuver, thruster 4 fired at a $40 \%$ dutycycle to maintain stability. As thruster 4 has a thrust component opposite the maneuver direction, the maneuver was lengthened on-board the spacecraft to compensate. An Astrogator simulation was performed to evaluate the effects of the dutycycle thrusting, the estimated attitude, and the adjusted maneuver time. This evaluation was performed by way of examining the downstream effects of the simulated maneuver on subsequent maneuvers.

\section{MATLAB - QuatGen}

Since MAP's on-board computer was not designed to generate the necessary control quaternions needed during maneuvers, ground processing was required to provide the attitude to MAP and the on-board computer was modified to accept this data. QuatGen was built by Rick Harman of NASA's Goddard Space Flight Center to meet this requirement. QuatGen was developed using MATLAB 5.1 and was run on a Windows NT platform. QuatGen inputs include spacecraft ephemeris data and solar ephemeris data. The spacecraft ephemeris can be from a NASA/GSFC Code 500 ephemeris, a STK ephemeris, or propagated from a Keplerian orbit state. Acceptable solar ephemeris inputs are a NASA/GSFC Code 500 Solar/Lunar/Planetary file or analytical from the Astronomical Almanac. The remaining inputs include the time span in question, the spacecraft attitude, and the frequency of quaternion output. Typically, QuatGen was executed to create data spanning from one hour prior to ignition to twelve hours after burnout. Data was created for a long postmaneuver period to ensure that sufficient attitude data was on-board the spacecraft in the event of a contingency (e.g. delaying the maneuver). The spacecraft attitude was entered in the form of an Eulersequence rotation. QuatGen was programmed to compute MAP's quaternions in the "Velocity-Sun" frame described above where a single $7.5^{\circ}$ pitch about the $\mathrm{Y}$-axis was required to provide the nominal attitude. In the event of a thruster failure, QuatGen (and Astrogator) could change to a two-thruster mode (using either thrusters $5 \& 6$ or $7 \& 8$ ) while choosing the appropriate pitch angle $-0^{\circ}$ or $15^{\circ}$, respectively. A new quaternion was computed for every 1 degree change in the velocity direction during the perigee pass. During MAP's phasing loops, this equated to a new quaternion every 30 seconds at perigee. A sample from the QuatGen graphical user interface (GUI) for data entry is 
shown in Figure 6. QuatGen was modified early in 2001 to account for the limits of the DSS field of view constraints. If, by orbit geometry, the Sun was not in the field of view at the beginning of the desired time span, QuatGen would set the initial quaternion at the limit of the field of view ( $45^{\circ}$ off of the $Z$-axis). Then QuatGen kept this attitude constant until the field of view constraint was met by the passage of MAP through perigee. A sample of the QuatGen output, showing this capability, is shown in Figure 7.

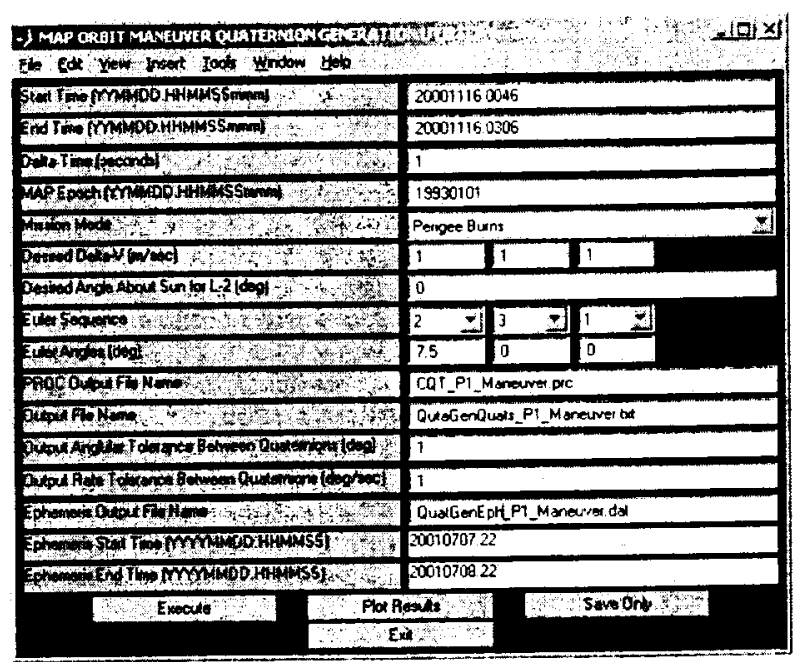

Figure 6: QuatGen Data Entry GUI

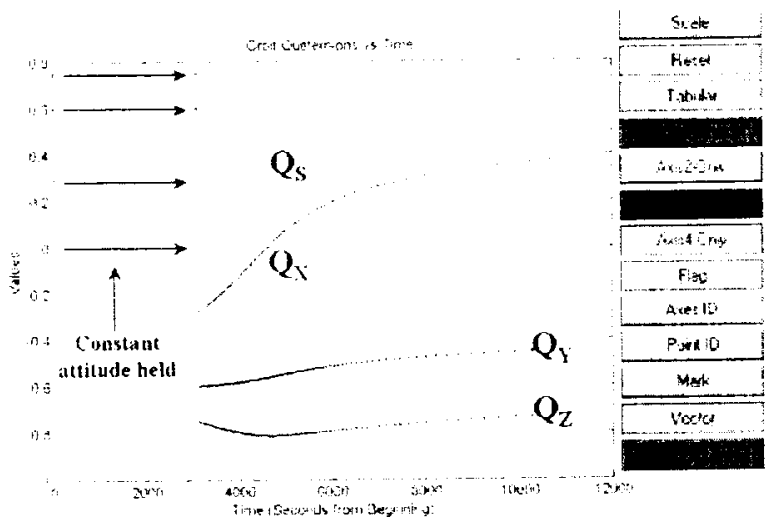

Figure 7: QuatGen Quaternion Plot

Three files are output from QuatGen. An ephemeris file (QuatGenEph) contains the spacecraft state (position \& velocity) and solar state (position only). A time history of the quaternions is contained in the QuatGenQuats file. The CQT file contains the quaternions in a format suitable for upload to MAP. These three files were delivered to the ACS engineers for inclusion in the $\mathrm{HiFi}$ and FlatSat simulations. All output files are written in simple ASCII text.

\section{Matrix-X - HiFi}

The ACS team built and maintains the High Fidelity (HiFi) simulator. $\mathrm{HiFi}$ is a software-only simulation of the spacecraft control system and dynamics, and was developed using the Matrix-X family of simulation and analysis tools. It includes sensor and actuator models, plus external force and torque models, including a simple orbit propagator. HiFi runs faster than real time, and can simulate any of the control modes and mode transitions. With the proper initialization and script files, multiple modes and maneuvers can be simulated, including multiple commanded attitudes and timed sequences of commands. The files needed to initialize the simulation, and the output of the simulation results, are described in the MAP Maneuver Operations Team Interface Control Document ${ }^{3}$. The HiFi simulation and the use of the Matrix-X tools in designing, analyzing, and simulating the MAP ACS is described in more detail in Reference 6 . The HiFi simulation can calculate the velocity impulse delivered to the spacecraft, but does not include a thruster blowdown model. The more accurate thruster model is left to Astrogator and to the FlatSat real time simulator.

There were initialization files set up for each maneuver to be performed, based on files previously created for running the $\mathrm{HiFi}$ simulation in support of software testing and simulation analysis. In addition, the Trajectory team provided ephemeris and attitude files, and a file containing maneuver start, duration, and initial condition information such as fuel tank pressure. A separate input script was written to read in these files and use their data to initialize the $\mathrm{HiFi}$ simulation. The timing of events in the simulation (like the de-spin from the compound spin science mode or the slew to the burn attitude) was controlled by manually modifying a part of the input script to match the timing in the ATS template for a given maneuver.

Once the simulation was run, separate MathScript files were run to create the necessary output files. There was a maneuver summary file that included information on duty cycles, estimated $\Delta V$ delivered, attitude errors, and burn duration. Also created were files containing the attitude profile and thruster command profile in a format that was readable by STK/Astrogator. Plots of sun angles, attitude errors, system momentum, and other relevant data were generated. These were FTP'd to a central location for use by the subsystem engineers for verification.

\section{FlatSat}

FlatSat is a real-time, hardware-in-the-loop Engineering Test Unit (ETU) flight software test platform. It is used to test interfaces to flight components, and to test the 
flight software in a realistic operations environment. The flight software is loaded to ETU's of the flight processors and flight boards, and interfaces with the ground command console. There is a Hybrid Dynamic Simulator (HDS) attached that simulates the spacecraft dynamics, sensors, actuators, and external environment. This facility was also used for acceptance-level flight software testing. For the MAP project, the control center consoles were hooked to FlatSat, and FlatSat was also used as a spacecraft mission simulator. Power subsystem racks were also integrated to FlatSat.

The interface to FlatSat was just like the flight operations interface, and all simulations had to be set up and run with flight-like procedures. The data was collected in sequentially printed output files, which were then post-processed with the $\mathrm{HiFi}$ simulation toolset (specifically, Xmath and custom-build data processing scripts) to generate plots and other data. Since FlatSat had ETU's of the flight processors and actual ground consoles, the ATS commands and the CQT needed for each maneuver, as well as the Failure Detection and Correction (FDC) configuration, could be tested to verify timing and configuration issues that aren't tested in $\mathrm{HiFi}$.

There were several files needed by FlatSat for a maneuver simulation to be run. The navigation team would take the trajectory data and the ATS template and generate a flight ATS for testing on FlatSat. In addition, for the long perigee maneuvers, the trajectory team generated CQT would be FTP'd to the FlatSat terminal for use in the test, after the navigation team had verified the format. The HiFi initialization file was also sent to the FlatSat terminal to be used for initialization of the HDS. A procedure was written to initialize and start the HDS and FlatSat. This script also read and loaded the ATS and the CQT to the ETU spacecraft processor. After the initial values were verified, the ATS would be enabled, and the test would nun in real time. This would verify the flight software configuration and timing, the commanded attitude profile, and also the effects of the pressure blowdown during the maneuver. All processor and HDS data were captured in sequential print files by the ground system.

At the end of the test, the data files would be FTP'd to the HiFi machine, where they could be ingested, plotted, and analyzed using script files generated for software testing. The output files were the same as for the HiFi simulation. These files were also sent to a central location for use by other subsystems. Most importantly, the attitude and thruster command profile files were fed back into STK/Astrogator to generate a "best estimate" prediction of the upcoming maneuver.

\section{Command Authorization Meeting}

The final step in the process was the Command Authorization Meeting (CAM). At this meeting, all the simulation results were presented to the project, including systems engineers, scientists, and other subsystems. The verification of predicted fuel use, maneuver duration, attitude, and $\Delta \mathrm{V}$ was shown, indicating good agreement between the trajectory, ACS, and propulsion subsystems. The contact timelines with the Deep Space Network (DSN) and the Tracking and Data Relay Satellite System (TDRSS) were discussed. Briefings on the current space weather and predicted radiation levels for the perigee passes were also included. In addition, an overall timeline, from loading the ATS and CQT to final post-maneuver safing of the propulsion system, was reviewed. Once the team was satisfied with the results, and understood the maneuver and possible contingencies and appropriate response, the subsystem lead engineers would sign an authorization form allowing the maneuver to proceed.

\section{Performance}

As mentioned above, one measure of performance for this process is to examine the errors induced downstream from feeding the attitude simulations back through Astrogator. In other words, what is the downstream $\Delta V$ penalty for the $\mathrm{P} 2$ and $\mathrm{P} 3$ maneuvers due to modeling errors at P1? This procedure was filtered downstream for each maneuver. The P2 maneuver induced an error in the P3 maneuver. The P3 maneuver induced an error in the PfCM while the $\mathrm{PfCM}$ induced an error in the MCC. In Table 2, the downstream induced errors are shown for each of the phasing loop maneuvers. In all cases, the error is small, less than $30 \mathrm{~cm} / \mathrm{s}$. The $\mathrm{P} 1$ and $\mathrm{P} 2$ induced errors are small because their corrections are being made at subsequent perigees (the optimal location for energy changes in an orbit). The error induced from P3 is added at the PfCM, executed 18 hours after perigee. This location is much less efficient than executing a maneuver at perigee. The induced error from PfCM (corrected at MCC, 7 days after lunar encounter) is largely due to the amplifying effect of the lunar encounter ${ }^{5}$. A discussion of the observed (calibrated) execution errors is also a way to evaluate the performance of the process. However, the observed errors contain the actual propulsion system performance as well as any orbit determination uncertainties and this goes beyond the modeling described above. As it were, this calibrated performance for the MAP system for all maneuvers was well within $5 \%$. This result was computed using a thrust scale factor in order to fit the observed data to the predicted data. 
Table 2: Performance of Planning Process

\begin{tabular}{|c|c|c|}
\hline Maneuver & $\begin{array}{c}\text { HiFi Induced } \\
\text { Error } \\
(\mathbf{c m} / \mathbf{s})\end{array}$ & $\begin{array}{c}\text { FlatSat Induced } \\
\text { Error } \\
\text { (cm/s) }\end{array}$ \\
\hline $\mathrm{P} 1$ & 1.0 & 1.1 \\
\hline $\mathrm{P} 2$ & -1.5 & -1.5 \\
\hline $\mathrm{P} 3$ & 26.3 & 27.9 \\
\hline $\mathrm{PfCM}$ & 18.1 & 18.1 \\
\hline
\end{tabular}

\section{Conclusions}

The greatest measure of the success of this maneuver planning process is that MAP successfully passed the Moon on July 30,2001 , at an altitude of $5000 \mathrm{~km}$. This gravity assist allowed MAP to enter a small amplitude Lissajous orbit about L2 without any insertion maneuver. At this time, MAP has completed its second stationkeeping maneuver and is well on its way to a two year mission at $\mathrm{L} 2$. This was made possible by the use of the COTS tools described above (STK, MATLAB, Matrix-X) and the hard work and diligence of the MAP Maneuver Team. The authors would like to acknowledge the hard work by the Navigation team lead, Dale Fink (Computer Sciences Corporation), and the Propulsion subystem lead, Gary Davis (NASA/GSFC).

\section{References}

1. Cuevas, O., Newman, L., Mesarch, M., and Woodard, M., "An Overview Of Trajectory Design Operations For The Microwave Anisotropy Probe Mission", AIAA 2002-4425, Monterey, CA, August 2002.

2. Davis, G., "The MAP Propulsion System", AIAA 2002-4156, Indianapolis, IN, July 2002.

3. Andrews, S., Cuevas, O., Davis, G., Fink, D., "Microwave Anisotropy Probe Interface Control Document", MAP Project Document, January, 2001.

4. Harman, R., "Map Orbit Maneuver Command Quaternion Generator (QuatGen) User's Guide", NASA Goddard Space Flight Center, January, 2001.

5. Mesarch, M., Rohrbaugh, D., Schiff, C., "Contingency Planning for the Microwave Anisotropy Probe Mission", AIAA 2002-4427, Monterey, CA, August 2002.

6. Anthony, T., Andrews, S., and McComas, D., "Use of the MatrixX Tools for Design and Development of the Attitude Control System for the Microwave Anisotropy Probe", 1996. 